\title{
Mapping the spatial distribution of star formation in cluster galaxies at $z \sim 0.5$ with the Grism Lens-Amplified Survey from Space (GLASS)
}

\section{Benedetta Vulcani and The GLASS Team}

Kavli Institute for the Physics and Mathematics of the Universe (WPI), Todai Institutes for Advanced Study, the University of Tokyo, Kashiwa, Japan

\begin{abstract}
We present the first study of the spatial distribution of star formation in $z \sim 0.5$ cluster galaxies. The analysis is based on data taken with the Wide Field Camera 3 as part of the Grism Lens-Amplified Survey from Space (GLASS). We illustrate the methodology by focusing on two clusters (MACS0717.5+3745 and MACS1423.8+2404) with different morphologies (one relaxed and one merging) and use foreground and background galaxies as field control sample. The cluster+field sample consists of 42 galaxies with stellar masses in the range $10^{8}-10^{11} \mathrm{M}_{\odot}$, and star formation rates in the range $1-20 \mathrm{M}_{\odot} y r^{-1}$. In both environments, $\mathrm{H} \alpha$ is more extended than the rest-frame UV continuum in $60 \%$ of the cases, consistent with diffuse star formation and inside out growth. The $\mathrm{H} \alpha$ emission appears more extended in cluster galaxies than in the field, pointing perhaps to ionized gas being stripped and/or star formation being enhanced at large radii. The peak of the $\mathrm{H} \alpha$ emission and that of the continuum are offset by less than $1 \mathrm{kpc}$. We investigate trends with the hot gas density as traced by the X-ray emission, and with the surface mass density as inferred from gravitational lens models and find no conclusive results. The diversity of morphologies and sizes observed in $\mathrm{H} \alpha$ illustrates the complexity of the environmental process that regulate star formation.
\end{abstract}

Keywords. galaxies: clusters: general, galaxies: evolution, galaxies: structure

Data characterization We present a pilot study characterizing the spatial distribution of the $\mathrm{H} \alpha$ emission in cluster galaxies beyond the local universe based on WFC3-IR data drawn from the Grism Lens-Amplified Survey from Space (GLASS; GO-13459; PI: Treu, Schmidt et al. 2014, Treu et al. 2015, http://glass.physics.ucsb.edu ). The GLASS G102 data yield spatially resolved $\mathrm{H} \alpha$ fluxes for all star-forming galaxies in the core $(<1 \mathrm{Mpc})$ of 10 clusters at $z=0.31-0.69$, with an order of magnitude improvement in sensitivity compared to previous studies (e.g. Sobral et al. 2013). Each cluster is observed at two different position angles. These two orientations allow us to mitigate the impact of contamination from overlapping spectra, and reliably measure for the first time the relative position of the $\mathrm{H} \alpha$ emission with respect to the continuum.

We analyze two of the ten clusters in the GLASS sample. We selected MACS0717.5+ 3745 (hereafter MACS0717) and MACS1423.8+2404 (hereafter MACS1423) based on the following criteria. First, we required similar redshift, so as to minimize evolutionary effects and differences in the sensitivity/selection function $(z \sim 0.55)$. Second, we selected them to be in very different dynamical states (MACS1423 is relaxed, MACS0717 is an active merger), so as to span the range of expected environments. A homogeneous control field sample is obtained by selecting objects in the immediate foreground and background of the two clusters. We then select galaxies with visually detectedH $\alpha$ in emission. In total 


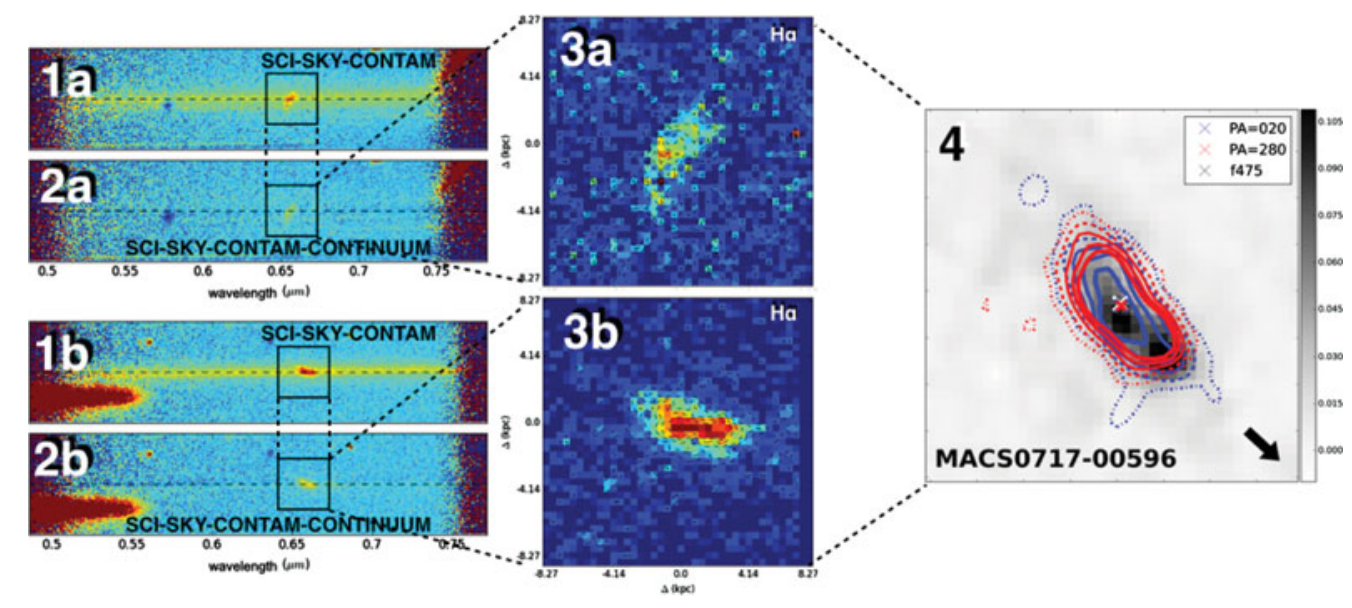

Figure 1. Example of the procedure followed to derive $H_{\alpha}$ maps. $a$ and $b$ refer to the two distinct PAs of the same galaxy. Panels $1 a$ and $1 b$ show the rest-frame flux-calibrated galaxy 2D spectra, after the sky background and the contamination have been subtracted. The dashed horizontal black lines show the y-position of the continuum. The box shows the position of the emission lines. Panels $2 a$ and $2 b$ show the rest-frame $2 \mathrm{D}$ spectra after the continuum has also been subtracted. Panels $3 a$ and $3 b$ show the map of the galaxy in the light of the $H_{\alpha}$ line. Panels 4 show the two maps over-plotted as contours on an image of the galaxy in the FW475 filter. Blue and red lines represent different levels of $H_{\alpha}$ flux. The arrows indicates the direction of the cluster center.

the sample presented in this pilot study consists of 42 objects, evenly distributed between the two clusters and the field (see Vulcani et al. 2015, for details).

\section{Methodology}

We have developed a new methodology to produce $\mathrm{H} \alpha$ maps taking advantage of the WFC3-G102 data at two orthogonal position angles. Indeed, the combined spatial resolution on the WFC3 and of the grism yield a spectrum that can be seen as images of a galaxy taken at $\sim 24 \AA$ increments $(\sim 12 \AA$ after interlacing $)$ and placed next to each other (offset by one pixel) on the detector. Thus, an emission feature in a high spatial resolution slitless spectrum is essentially an image of a galaxy at that wavelength.

The methodology is largely presented in Vulcani et al. (2015). Briefly, to create $\mathrm{H} \alpha$ emission line maps, we initially treat the spectra coming from the different exposures (one per PA) of each galaxy independently. We use the flux-calibrated galaxy 2D continuum spectra (Fig.1, panels $1 a$ and $1 b$ ), after the sky background and the contamination have been subtracted. From two regions contiguous to the $\mathrm{H} \alpha$ emission we determine the $y$ position of the peak of the continuum. We then measure the offset in the $y$-direction of the $\mathrm{H} \alpha$ emission with respect to the galaxy center in the light of the continuum. Subsequently, we subtract the 2D stellar continuum model obtained by convolving the best-fit 1D SED without emission lines with the actual 2D data, ensuring that all model flux pixels are non-negative (Fig.1, panels $2 a$ and $2 b$ ). We find that counts around the lines are slightly negative, suggesting that the continuum subtraction is somewhat too aggressive. Therefore, we measure the median flux in regions close to the emission line and further subtract it from the entire spectrum. The residual is a map of the galaxy in the light of the $\mathrm{H} \alpha$ line (Fig.1, panels $3 a$ and $3 b$ ). As a last step, we superimpose the $\mathrm{H} \alpha$ map onto an image of the galaxy taken with the F475W filter (rest-frame UV) (Fig.1, panel 4$)$. We use F475W to map relatively recent ( 100Myr) star formation, as opposed to ongoing ( $10 \mathrm{Myr})$ star formation traced by $\mathrm{H} \alpha$. To do so, we align each 
map to the image of the galaxy in the light of the continuum, rotating each map by the angle of its PA, keeping the $y$-offset unaltered with the respect to the continuum. On the $x$-axis, there is a degeneracy between the spatial dimension and the wavelength uncertainty, it is therefore not possible to determine very accurately the central position of the $\mathrm{H} \alpha$ map for each PA separately. Nonetheless, for the cases in which spectra from both PAs are reliable (28/42), we use the fact that the 2 PAs differ by almost 90 degree, therefore the $x$-direction of one spectrum roughly corresponds to the $y$-direction of the second spectrum and vice-versa. We can therefore shift the two spectra independently along their $x$-direction to force the center of the emission of the two maps to coincide.

For the galaxies with reliable spectra in both PAs, we can also measure the real distance between the peak of the $\mathrm{H} \alpha$ emission and the continuum emission, obtained as the quadratic sum of the two offsets.

Finally, for the cluster galaxies, we also measure the offset between the $\mathrm{H} \alpha$ and the continuum as projected along the cluster radial direction, determined by the line connecting the clustercenter and the galaxy center in the continuum light. We assign a positive sign to the projected offset when the peak of the $\mathrm{H} \alpha$ is between the cluster center and the peak of the continuum.

Sizes have been obtained by computing the second order moment of the light distribution, which gives us the width of the distribution and therefore the extension of the galaxy, and corrected for the point spread function of our observations.

\section{Results}

\section{Maps of $H \alpha$ and continuum emission}

We take the ratio of the average size as measured from the $\mathrm{H} \alpha$ light $(\mathrm{r}(\mathrm{H} \alpha))$ to the average size as measured from the rest-frame UV continuum $(\mathrm{r}(\mathrm{F} 745 \mathrm{~W}))$. The F745W continuum traces the star formation occurred over the last $100 \mathrm{Myr}$, so that we compare the currently star forming regions to the less recent ones. We note that similar results are obtained when using the observed F110W filter, which traces the bulk of the older stellar population.

In both environments, distributions of the ratios peak around 1, showing that many galaxies have comparable sizes in $\mathrm{H} \alpha$ and continuum. However, distributions are slightly skewed toward values $>1$. In cluster galaxies mean size ratios are systematically larger than field galaxies: mean values are driven by a subpopulation of galaxies $(\sim 20 \%)$ that present $\mathrm{H} \alpha$ emission at least two-three time as extended as the light in the continuum. No such examples are present in the field. This might suggest that in all environments the ongoing star formation is occurring over a larger area than that of the recent one, but in clusters there might be some additional mechanisms that are stripping the gas and star formation is continuing in the stripped material.

We group galaxies according to their $\mathrm{r}(\mathrm{H} \alpha) / \mathrm{r}(\mathrm{F} 745 \mathrm{~W})$. The great majority of cluster and field galaxies (15/25 and 10/17 respectively) present size of the $\mathrm{H} \alpha$ light larger than the size measured from the continuum $(\mathrm{r}(\mathrm{H} \alpha) / \mathrm{r}(\mathrm{F} 745 \mathrm{~W})>1.2)$. Though being star forming, most of these galaxies show an early-type morphology in the color composite images. In clusters, this might be a sign of ongoing stripping. 6/25 cluster and 3/17 field galaxies have similar sizes in $\mathrm{H} \alpha$ and in the continuum, while the rest of the galaxies have $\mathrm{H} \alpha$ sizes smaller than continuum sizes. In general, our sample includes galaxies with a variety of morphologies and there is no clear correlation between the extent of the $\mathrm{H} \alpha$ emission and the galaxy color or morphology. This might suggest that there is no a unique mechanism responsible for extension of the $\mathrm{H} \alpha$, but that different processes might be at work in galaxies of different types.

We also observe that the peak of the $\mathrm{H} \alpha$ emission is displaced with respect to the F475W continuum emission. In both environments, the displacement is always smaller 
than 1kpc. Cluster galaxies might be characterized by a marginally larger offset than field galaxies, but a larger number statistics will be needed to confirm the trends. The existence of the offset suggests that in most galaxies the bulk of the ongoing star formation is not occurring in the galaxy cores.

Maps of $\mathrm{H \alpha}$ and position within the clusters

For cluster galaxies, we correlate their $H_{\alpha}$ morphology with their location in the cluster, the surface mass density distribution and the X-ray emission. In both clusters, galaxies are located within $\sim 0.4 \mathrm{r}_{500}$ and do not seem to avoid the cluster cores, even though there might be possible projection effects. The two clusters present very different surface mass density distribution and X-ray emission: MACS0717 has more than one main peak in the emissions, MACS1423 shows a symmetric surface mass density distribution and X-ray emission. In MACS0717 we find galaxies with both truncated or extended $\mathrm{H} \alpha$ with respect to the continuum, in MACS1423 all galaxies have $\mathrm{H} \alpha$ light more extended. Despite the small number statistics, it appears that the truncated objects are only found between the merging clusters, suggesting that the spatial distribution of $\mathrm{H} \alpha$ is indeed related to cluster dynamics: the most extreme cases of stripping are expected to take place in interacting systems (e.g., Owers et al. 2012).

We note that the lack of strong correlations does not allow us to identify a unique strong environmental effect that originates from the cluster center. We hypothesize that local effects, uncorrelated to the cluster-centric radius, play a larger role. Such effects weaken potential radial trends.

\section{Conclusions}

A complex picture emerges and a simple explanation can not describe our observations. Even though galaxies in clusters and in the field present similar $\mathrm{H} \alpha$ properties, the variety of their morphologies suggests that they are at different stages of their evolution, therefore there can not be a unique mechanism acting on galaxies in the different environments.

The larger extension of $\mathrm{H} \alpha$ with respect to the continuum seems to be a generic indication of inside-out growth (see also Nelson et al. 2012). However, given the variety of morphologies, well-ordered disks do not appear to be the only site of star formation.

The larger relative extension of $\mathrm{H} \alpha$ in clusters than in the field point to additional cluster-specific mechanisms responsible for stripping the ionized gas or perhaps for triggering additional star formation in the outskirts of the galaxies, such as ram pressure stripping of the ionized gas and/or tidal compression of the outskirts or the galaxies.

In few cases, the smaller $\mathrm{H} \alpha$ disk than the existing stellar population suggests that galaxies have been already deprived from their gas.

An ongoing extended analysis will allow us to further distinguish and classify the processes acting in clusters from those acting on the field, and to better correlate galaxy properties with the cluster global properties, investigating in detail the role of the environment in shutting down star formation.

\section{References}

Nelson et al., 2012, ApJL, 747, L28

Owers, et al., 2012, ApJL, 750, L23.

Schmidt et al., 2014, ApJ, 782, L36

Schmidt et al., 2015, ApJ submitted

Sobral et al., 2013, MNRAS, 428, 1128

Treu et al., 2015, ApJ in press, arXiv:astro-ph:1509.00475

Vulcani et al., 2015, ApJ submitted 